\title{
Internal supersymmetry and small-field Goldstini
}

\author{
Diederik Roest, ${ }^{a}$ Pelle Werkman ${ }^{a}$ and Yusuke Yamada ${ }^{b}$ \\ ${ }^{a}$ Van Swinderen Institute for Particle Physics and Gravity, University of Groningen, \\ Nijenborgh 4, 9747 AG Groningen, The Netherlands \\ ${ }^{b}$ Stanford Institute for Theoretical Physics and Department of Physics, Stanford University, \\ 382 Via Pueblo Mall Stanford CA 94305, U.S.A. \\ E-mail: d.roest@rug.nl, p.j.werkman@rug.nl, yusukeyy@stanford.edu
}

ABSTRACT: The dynamics of the Goldstino mode of spontaneously broken supersymmetry is universal, being fully determined by the non-linearly realized symmetry. We investigate the small-field limit of this theory. This model non-linearly realizes an alternative supersymmetry algebra with vanishing anti-commutators between the fermionic generators, much like an internal supersymmetry. This Goldstino theory is akin to the Galilean scalar field theory that arises as the small-field limit of Dirac-Born-Infeld theory and non-linearly realizes the Galilean symmetry. Indeed, the small-field Goldstino is the partner of a complex Galilean scalar field under conventional supersymmetry. We close with the generalization to extended internal supersymmetry and a discussion of its higher-dimensional origin.

KEYWORds: Extended Supersymmetry, Space-Time Symmetries, Superspaces, Supersymmetry Breaking

ARXIV EPRINT: 1710.02480 


\section{Contents}

1 Introduction 1

2 The internal supersymmetry algebra and Goldstino 3

3 Adding linear supersymmetry and Galileons 5

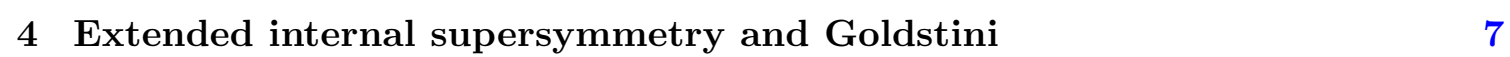

$\begin{array}{llr}5 & \text { Conclusions } & 8\end{array}$

\section{Introduction}

Symmetries in their various guises form the cornerstone of modern physics. Of particular interest is the case of spontaneously broken symmetries, either of internal $[1,2]$ or space-time nature [3]. Both cases lead to Goldstone modes that transform in a non-linear representation. The resulting Goldstone dynamics is characterized by a small number of coefficients and therefore has clear signatures, including masslessness at quadratic order and special soft limits at higher orders [4-6].

A beautiful example is provided by a scalar in $D=4$ Poincaré invariant field theories. The scalar can arise as a Goldstone boson from various extensions of the space-time symmetry, including the well-known possibilities of $D=4$ conformal and $D=5$ Poincaré. The allowed interactions for these scalar fields have been constructed from probe brane constructions $[7,8]$. For Poincaré, this includes the Dirac-Born-Infeld (DBI) action and its higher-derivative versions. These are invariant under

$$
\delta \phi=c+b_{\mu}\left(x^{\mu}+\phi \partial^{\mu} \phi\right)
$$

corresponding to the translation $P_{5}$ and the rotation $M_{\mu 5}$ of the higher-dimensional algebra.

The third possibility arises as a singular contraction of the Poincaré (or conformal) algebra, defined as

$$
P_{5} \rightarrow \omega P_{5}, \quad M_{\mu 5} \rightarrow \omega M_{\mu 5}, \quad \text { with } \omega \rightarrow \infty .
$$

It preserves the $D=4$ Poincaré part, and in addition has

$$
\begin{aligned}
{\left[P_{5}, M_{\mu 5}\right] } & =0, \quad\left[P_{\mu}, M_{\nu 5}\right]=i \eta_{\mu \nu} P_{5}, \\
{\left[M_{\mu \nu}, M_{\rho 5}\right] } & =i\left(\eta_{\mu \rho} M_{\nu 5}-\eta_{\nu \rho} M_{\mu 5}\right) .
\end{aligned}
$$

The resulting Galilean algebra differs from the $D=5$ Poincaré algebra only in the first commutator being zero. Moreover, it is a non-trivial extension of the space-time group due 
to the non-vanishing of the second commutator; the final commutator just expresses the Lorentz nature of the additional vector generator.

The same limit can be performed on the field theory side. DBI can be written as a coset in terms of the combination $\phi P_{5}$ and is invariant under (1.2) with

$$
\phi \rightarrow \phi / \omega, \quad \text { with } \omega \rightarrow \infty
$$

In this singular, small-field limit, one obtains an inequivalent coset that is based on the contracted, Galilean algebra. The non-linear transformation becomes [7]

$$
\delta \phi=c+b_{\mu} x^{\mu}
$$

and hence is field-independent. The leading interactions for this theory, referred to as Galileons, are Wess-Zumino terms [9]. The cubic one describes the brane bending mode of the DGP model [10], and more generally these interactions describe models of massive gravity in their decoupling limits [11]. Moreover, their covariantized version [12] has been used to describe late-time acceleration in cosmology; however, this particular setup may be ruled out by recent observations [13].

The above three theories with enhanced (Poincaré, conformal or Galilean) symmetry are unique in having a special soft limit of the amplitudes [4-6]; this is intimately tied to the extended and non-linear symmetries of these particular theories.

One might wonder whether there is a similar pattern on the fermionic side, with Grassmannian extensions $Q_{\alpha}$ of the Poincaré algebra. Indeed such a construction is possible for $\mathcal{N}=1$ supersymmetry, leading to the Volkov-Akulov (VA) Goldstino with transformation $[14-16]$

$$
\delta \lambda^{\alpha}=\epsilon^{\alpha}-i\left(\lambda \sigma^{\mu} \bar{\epsilon}-\epsilon \sigma^{\mu} \bar{\lambda}\right) \partial_{\mu} \lambda^{\alpha}
$$

Similar to the bosonic construction, this theory can be interpreted as a three-brane in superspace [18] and has an enhanced soft limit as compared to generic interacting fermion theories [19].

Could there be different supersymmetry algebras ${ }^{1}$ leading to inequivalent Goldstino dynamics? We will demonstrate that this is indeed the case and construct an alternative, which we will refer to as internal supersymmetry. It acts on the corresponding Goldstino mode as a constant shift, allowing for specific interactions only (see section 2). Such a symmetry appears in the limit where the corresponding Goldstino (super)field fluctuation is very small: the Goldstino describes small fluctuations of a supersymmetric brane, which (partially) breaks supersymmetry as well as Poincaré symmetry in four (or higher) dimensions.

Under conventional linear supersymmetry, the non-linear transformation laws of fermions and bosons are related. Indeed this happens for the field-dependent transformations (1.1) and (1.6), which are invariances of $\mathcal{N}=1$ super-DBI theory constructed

\footnotetext{
${ }^{1}$ This might appear to be ruled out by the superalgebra classification of [20]. However, similar to the Coleman-Mandula theorem on extensions of space-time symmetry [21], this only concerns superalgebras with linear representations.
} 
in [22-25]. Phrased differently, this theory has non-linearly realized Poincaré and supersymmetry generators that commute with linear $D=4$ super-Poincaré into $D=6$ super-Poincaré, and the non-linearly realized generators commute into each other under the linearly realized supersymmetry. A natural question regards the non-linear symmetries of this theory in the small-field limit. We will demonstrate that these become the Galilean symmetry and internal supersymmetry, which again are related under linear supersymmetry (see section 3 ).

We further generalize our construction by considering the appropriate non-relativistic contraction of the $D=10$ supersymmetry algebra instead. This leads to a relativistic $D=4$ theory with linearly realized minimal supersymmetry, as well as non-linearly realized $\mathcal{N}$-extended internal supersymmetry (see section 4 ).

\section{The internal supersymmetry algebra and Goldstino}

To demonstrate how one can obtain the alternative supersymmetry algebra by means of a simple contraction, we start with the $\mathcal{N}=1$ super-Poincaré algebra in $^{2} D=4$, with fermionic generators $Q_{\alpha}$ subject to

$$
\left\{Q_{\alpha}, \bar{Q}_{\dot{\beta}}\right\}=-2 \mathrm{i} \sigma_{\alpha \dot{\beta}}^{\mu} P_{\mu}, \quad\left[M_{\mu \nu}, Q_{\alpha}\right]=\left(\sigma_{\mu \nu} Q\right)_{\alpha} .
$$

Under the İnönü-Wigner contraction

$$
Q_{\alpha} \rightarrow \omega S_{\alpha}, \quad \text { with } \omega \rightarrow \infty
$$

where we have relabelled the supercharge generator as $S$ to emphasize its physical difference, this algebra becomes

$$
\left\{S_{\alpha}, \bar{S}_{\dot{\beta}}\right\}=0, \quad\left[M_{\mu \nu}, S_{\alpha}\right]=\left(\sigma_{\mu \nu} S\right)_{\alpha} .
$$

Finally, the supersymmetry generators have a non-trivial weight under a U(1) R-symmetry.

Note that the hallmark of the supersymmetric extension of the Poincare space-time symmetry group, i.e. that supercharges anti-commute into translations, has disappeared in this limit. The only non-trivial commutator is with Lorentz generators, reflecting the fact that $S$ transforms as spin-1/2. This fermionic extension of Poincaré is akin to an internal symmetry, and we will refer to it as internal supersymmetry.

The above algebra contraction can be seen as the small-field limit of the $\mathcal{N}=1$ super-Poincaré theory. Again the coset combination $\lambda^{\alpha} Q_{\alpha}$ is invariant under the algebra contraction together with the fermion small-field limit

$$
\lambda^{\alpha} \rightarrow \lambda^{\alpha} / \omega, \quad \text { with } \omega \rightarrow \infty .
$$

In this limit, the original VA transformation of the Goldstino $\lambda^{\alpha}$ (1.6) yields a simple fermionic shift,

$$
\delta \lambda^{\alpha}=\epsilon^{\alpha},
$$

where $\epsilon^{\alpha}$ is a constant parameter.

\footnotetext{
${ }^{2}$ For definiteness of fermionic conventions, we will focus on $D=4$, but much of our discussion carries over to other dimensions.
} 
Note the clear analogy between the field-dependent transformations of DBI (1.1) and VA (1.6), and their small-field limits (1.5) and (2.5): in both cases one loses the field dependence in the small-field limit, corresponding to the vanishing of the crucial first commutators in (1.3) and (2.3). One would expect the contracted algebras to only have non-linear representation, and internal supersymmetry therefore cannot be restored as a linear transformation above some energy scale. Indeed, this theory has been shown not to admit unitary UV completions [26], similar to Galileons; instead, it could provide an alternative realization of fermion compositeness with specific LHC signatures, as discussed in $[27,28]$.

Turning to invariants, the lowest order invariant (up to a total derivative) is the simple fermionic kinetic term,

$$
i \bar{\lambda} \sigma^{\mu} \partial_{\mu} \lambda
$$

which arises as the small field limit of the VA invariant [14-16]. While the latter includes interactions, the theory becomes free in the small-field limit. ${ }^{3}$

We can see that further Wess-Zumino terms do not exist in $D=4$ by using the coset formalism going back to $[1,2]$. The invariant 1-forms appearing in the decomposition of the Maurer-Cartan form are the following:

$$
\omega_{P}^{\mu}=d x^{\mu}, \quad \omega_{S}^{\alpha}=d \lambda^{\alpha},
$$

where $\lambda$ is Majorana. The Wess-Zumino terms are obtained by wedging these 1-forms together to a 5 -form living in a space in which $\lambda^{\alpha}$ is promoted to a coordinate. This form must then be pulled back to the four-dimensional space defined by $\lambda=\lambda(x)$. Wedging together the 1-forms immediately implies anti-symmetrization of all derivatives of $\lambda$ appearing in a Wess-Zumino term. The possibilities for quartic interaction terms are then highly limited by Lorentz invariance. All possible terms are of the following form:

$$
(\bar{\lambda} \gamma \partial \lambda)(\partial \bar{\lambda} \gamma \partial \lambda)
$$

where $\gamma$ denotes a gamma matrix of some rank. Both gamma matrices must be rank one or two, since other choices are immediately vanishing or total derivative due to Majorana flip relations. The only possible terms are then:

$$
\left(\bar{\lambda} \gamma^{\mu_{1}} \partial_{\left[\nu_{1}\right.} \lambda\right)\left(\partial_{\nu_{2}} \bar{\lambda} \gamma^{\mu_{2} \mu_{3}} \partial_{\left.\nu_{3}\right]} \lambda\right) .
$$

with some product of Kronecker deltas and Levi-Civita tensors contracting the indices. However, each term is trivial due to a Fierz identity. It therefore appears that, at least in $D=4$, the situation for fermions is akin to that of vectors, for which a no-go theorem for Galileon-like interactions was proven in [17]. It would be interesting to investigate whether non-trivial Wess-Zumino terms exist in dimensions higher than 4, where the structure of Fierz identities and Majorana flips is different and Wess-Zumino terms beyond quartic order in fermions can exist.

\footnotetext{
${ }^{3} \mathrm{An}$ analogous story can be found on the bosonic side, where the lowest-order invariant becomes the free kinetic theory in the Galilean limit, while higher-order invariants introduce cubic and quartic interactions.
} 
In the absence of Wess-Zumino terms, the fermion is derivatively coupled in all invariants, which implies that they give rise to second-order field equations and Ostrogradsky instabilities. In some cases, however, a supersymmetric coupling to a healthy bosonic sector can remove the instability, as the example of the next section demonstrates.

\section{Adding linear supersymmetry and Galileons}

Our second goal will be to show that internal supersymmetry can be combined with a linearly realized supersymmetry of conventional nature, under which it is the natural partner of Galilean transformations. A useful starting point will be the minimal supersymmetry algebra in $D=6$, which has eight supercharges and $\mathrm{SU}(2)$ Majorana-Weyl spinors (see e.g. [29]). Rewriting this algebra in terms of $D=4$ Weyl spinors via

$$
Q_{1 \underline{\alpha}}=\left(Q_{\alpha},-\bar{S}^{\dot{\alpha}}\right)^{T}, \quad Q_{2 \underline{\alpha}}=\left(S_{\alpha}, \bar{Q}^{\dot{\alpha}}\right),
$$

where $\underline{\alpha}$ is a 4-component spinor index of the SU(2) Majorana-Weyl spinor, the anticommutators of the supercharges read

$$
\begin{aligned}
& \left\{Q_{\alpha}, \bar{Q}_{\dot{\beta}}\right\}=\left\{S_{\alpha}, \bar{S}_{\dot{\beta}}\right\}=-2 \mathrm{i} \sigma_{\alpha \dot{\beta}}^{\mu} P_{\mu}, \\
& \left\{Q_{\alpha}, S_{\beta}\right\}=2 \epsilon_{\alpha \beta} P_{z}, \quad\left\{\bar{Q}^{\dot{\alpha}}, \bar{S}^{\dot{\beta}}\right\}=-2 \epsilon^{\dot{\alpha} \dot{\beta}} P_{\bar{z}},
\end{aligned}
$$

where $z=\frac{1}{2}\left(x^{4}-\mathrm{i} x^{5}\right)$ and $P_{z}=P_{4}+\mathrm{i} P_{5}$ in our conventions. This is the $\mathcal{N}=2$ extended supersymmetry algebra in $D=4$, with $\mathrm{U}(2)$ R-symmetry group and $\mathrm{SO}(1,5)$ automorphisms inherited from its six-dimensional origin.

We now consider the Galilean rescaling (1.2) of this algebra. Importantly, this limit would be incompatible without scaling the fermions as well: the anti-commutator would become singular. Therefore there is no $\mathcal{N}=2$ extension of the Galilean algebra with the usual supersymmetry. Instead, the interesting and consistent option is to rescale one component of the $\mathrm{U}(2)$ doublet, which we take to be $S$ without loss of generality:

$$
Q_{\alpha} \rightarrow Q_{\alpha}, \quad S_{\alpha} \rightarrow \omega S_{\alpha}, \quad \text { with } \omega \rightarrow \infty,
$$

in addition to the rescalings (1.2) of the bosonic generators $P_{z}$ and $M_{\mu z}$. In addition, the off-diagonal generators of SU(2), denoted by $R$ and its conjugate, both scale as

$$
R \rightarrow \omega R, \quad \bar{R} \rightarrow \omega \bar{R}, \quad \text { with } \omega \rightarrow \infty,
$$

for consistency.

After the contraction, the anti-commutators between the different supersymmetry generators are

$$
\begin{aligned}
& \left\{Q_{\alpha}, \bar{Q}_{\dot{\beta}}\right\}=-2 \mathrm{i} \sigma_{\alpha \dot{\beta}}^{\mu} P_{\mu}, \quad\left\{S_{\alpha}, \bar{S}_{\dot{\beta}}\right\}=0, \\
& \left\{Q_{\alpha}, S_{\beta}\right\}=-2 \epsilon_{\alpha \beta} P_{z}, \quad\left\{\bar{Q}^{\dot{\alpha}}, \bar{S}^{\dot{\beta}}\right\}=2 \epsilon^{\dot{\alpha} \dot{\beta}} P_{\bar{z}},
\end{aligned}
$$


where only the second anti-commutator differs from the original algebra. Secondly, Lorentz boosts in the contracted directions satisfy

$$
\left[M_{\mu \bar{z}}, Q_{\alpha}\right]=\frac{1}{2} \mathrm{i}\left(\sigma_{\mu} \bar{S}\right)_{\alpha}, \quad\left[M_{\mu \bar{z}}, S_{\alpha}\right]=0 .
$$

The final set of rescaled generators has commutators

$$
\left[R, Q_{\alpha}\right]=S_{\alpha}, \quad\left[R, S_{\alpha}\right]=0
$$

Finally, this algebra inherits two copies of U(1) from its original R-symmetry group.

This algebra can be seen as an extension of the usual $\mathcal{N}=1$ super-Poincare algebra with the rescaled Galilean-like generators, all of which are realized non-linearly. Note that they form a sequence under linear supersymmetry: $M_{\mu z}$ and $R$ transform into $S$ under $Q$, which in turn is transformed to ${ }^{4} P_{z}$ :

$$
Q_{\alpha}: \quad\left(M_{\mu z}, R\right) \rightarrow S_{\alpha} \rightarrow P_{z} \rightarrow 0,
$$

Moreover, this extension is not fully internal due to the non-trivial commutator of $M_{\mu z}$ with translations.

Turning to realizations, a natural formulation of the non-linear symmetries of this theory presents itself in terms of a superfield of the linear supersymmetry. Consider a chiral superfield $\Phi$ given by

$$
\Phi=\phi+\lambda \theta+F \theta \theta
$$

consisting of a complex scalar field $\phi$, a fermion $\lambda$ as well as an auxiliary field $F$. Under linear supersymmetry, these components transform as

$$
\delta \phi=\bar{\epsilon} \lambda, \quad \delta \lambda=\bar{\epsilon} \sigma^{\mu} \partial_{\mu} \phi+F \epsilon \quad \delta F=\bar{\epsilon} \sigma^{\mu} \partial_{\mu} \lambda .
$$

Moreover, the Galileon-like generators (3.8) act on this superfield as

$$
\Phi \rightarrow \Phi+c+\theta \eta+b_{\mu}\left(x^{\mu}+i \theta \sigma^{\mu} \bar{\theta}\right)+f \theta \theta,
$$

or equivalently,

$$
\phi \rightarrow \phi+c+b_{\mu} x^{\mu}, \quad \lambda_{\alpha} \rightarrow \lambda_{\alpha}+\eta_{\alpha}, \quad F \rightarrow F+f,
$$

with constant parameters, corresponding to the generators $P_{z}, M_{\mu z}, S_{\alpha}$ and $R$, respectively. Importantly, these all preserve the chiral nature of the superfield.

Finally, the invariant Lagrangians can be written in terms of superfields. At lowest order, the ordinary kinetic terms follow from the usual superspace expression $\Phi \bar{\Phi}$, which includes the Dirac action (2.6) for the fermionic component. The first interactions, at the quartic level, can be classified according to which of the generators in the sequence (3.8) are realized. Its smallest part is the bosonic shift symmetry with the invariant $D \Phi D \Phi \bar{D} \bar{\Phi} \bar{D} \bar{\Phi}$,

\footnotetext{
${ }^{4} \mathrm{~A}$ possible further extension of this sequence would be the special Galileon symmetry [30] of a real scalar field; however, no such symmetry is known for the complex case.
} 
including the purely bosonic term $(\partial \phi)^{4}$ at mass dimension-8, see e.g. [31]. The entire sequence is realized by ${ }^{5}[33]$ :

$$
\Phi\left(\bar{D}_{\dot{\alpha}} \partial_{\mu} \bar{\Phi} \bar{\sigma}_{\nu}^{\dot{\alpha} \alpha} D_{\alpha} \partial_{\rho} \Phi\right) \epsilon^{\mu \nu \rho \sigma} \partial_{\sigma} \bar{\Phi} .
$$

This is the first non-trivial Wess-Zumino term for the sequence (3.8). This interaction plays a similar role to the purely bosonic Galileon, as it describes the fluctuations of a brane in superspace, after taking the small-field limit of the bending modes and the Goldstino. When truncated to the complex scalar, ${ }^{6}$ it is proportional to the usual quartic Galileon at mass dimension-10:

$$
\phi\left(\partial_{\mu_{1}} \partial^{\left[\mu_{1}\right.} \phi\right)\left(\partial_{\mu_{2}} \partial^{\mu_{2}} \bar{\phi}\right)\left(\partial_{\mu_{3}} \partial^{\left.\mu_{3}\right]} \bar{\phi}\right) .
$$

At the same order, the fermionic contribution reads

$$
-4 i \partial_{\mu} \lambda \sigma^{\tau} \partial_{\tau} \bar{\lambda} \partial_{\sigma} \bar{\lambda} \bar{\sigma}_{\nu} \partial_{\rho} \lambda \epsilon^{\mu \nu \rho \sigma}+-2 i \partial_{\mu} \bar{\lambda} \bar{\sigma}_{\nu} \sigma^{\kappa} \partial_{\sigma} \bar{\lambda} \epsilon^{\mu \nu \rho \sigma} \partial_{\kappa} \lambda \partial_{\rho} \lambda
$$

While these terms are manifestly invariant under internal supersymmetry, this is not the case for the mixed scalar-fermion terms, which can be found in [33]; however, one can check that these always multiply total derivatives and hence do not affect the field equations.

By construction, the bosonic field equations have the Galileon structure and hence do not propagate any ghosts. The fermionic sector, however, necessarily has second-order field equations and hence seems to propagate a ghost. This seems a paradoxical conclusion, as the theory is also linearly supersymmetric and hence cannot have purely fermionic ghosts. We believe this to be resolved by the coupling between the bosons and fermions of this theory, along the lines of [35-37].

\section{Extended internal supersymmetry and Goldstini}

Finally, we will demonstrate that it is possible to have $\mathcal{N}$-extended internal supersymmetry, in addition to linearly realized supersymmetry. Instead of taking $D=6$ as a starting point, one can perform a similar analysis for $D=10$ minimal supersymmetry. Its supersymmetry generator is a Majorana-Weyl spinor that decomposes into four 4D Majorana spinors $Q_{i}$ with $i=1, \ldots, 4$. As in the $6 \mathrm{D}$ case, the rotation in the extra dimensions becomes part of the R-symmetry, spanning the adjoint $\mathbf{1 5}$ of $\mathrm{SO}(6) \simeq \mathrm{SU}(4)$. The supersymmetry generators $Q_{i}$ transform in the fundamental of $\mathrm{SU}(4)$, while the translations $P_{m}$ as well as the Lorentz transformations $M_{\mu m}$ with $m=4, \ldots, 9$ form a 6 self-dual representation.

In analogy with the $6 \mathrm{D}$ situation, the contraction of the algebra requires the decomposition of SU(4) into $\mathrm{SU}(3) \times \mathrm{U}(1)$, with $\mathbf{4} \rightarrow \mathbf{3}+\mathbf{1}$ and $\mathbf{6} \rightarrow \mathbf{3}+\overline{\mathbf{3}}$ or in terms of indices $i=(I, 4)$ and $m=(I, \bar{I})$. We then perform the scaling

$$
\begin{aligned}
P_{I} & \rightarrow \omega P_{I}, & M_{\mu I} & \rightarrow \omega M_{\mu I}, \\
Q_{I \alpha} & \rightarrow \omega S_{I \alpha}, & \text { with } \omega & \rightarrow \infty,
\end{aligned}
$$

\footnotetext{
${ }^{5}$ At the cubic level in $\Phi$, there are no Wess-Zumino terms of (3.8) which contain a Galileon term. See [32].

${ }^{6}$ An analogous construction for a $D=3$ superfield containing a real scalar can be found in [34].
} 
where $I=1,2,3$. Similar to the previous section, this is not sufficient for a consistent contraction as the commutator between $Q \equiv Q_{4}$ and some of the $M_{m n}$ diverges. One needs to split up the R-symmetry generators as well, with $\mathbf{1 5} \rightarrow \mathbf{8}+\mathbf{3}+\overline{\mathbf{3}}+\mathbf{1}$. We denote the $\mathbf{3}$ and $\overline{3}$ generators as $R_{I}$ and $\bar{R}_{\bar{I}}$, and rescale both similar to (3.4).

The resulting algebra takes the following form. In addition to conventional supersymmetry with generators $Q$, it contains internal supersymmetry generators $S_{I \alpha}$ with

$$
\begin{aligned}
\left\{Q_{\alpha}, \bar{Q}_{\dot{\beta}}\right\} & =-2 \mathrm{i} \sigma_{\alpha \dot{\beta}}^{\mu} P_{\mu}, \quad\left\{S_{I \alpha}, \bar{S}_{\bar{J} \dot{\beta}}\right\}=0, \\
\left\{Q_{\alpha}, S_{I \beta}\right\} & =-2 \epsilon_{\alpha \beta} P_{I}, \quad\left\{\bar{Q}^{\dot{\alpha}}, \bar{S}_{\bar{I}}^{\dot{\beta}}\right\}=2 \epsilon^{\dot{\alpha} \dot{\beta}} P_{\bar{I}} .
\end{aligned}
$$

Here we have defined $P_{I}=P_{2+2 I}+\mathrm{i} P_{3+2 I}$, which together with $M_{\mu I}$ are the Galilean transformations that form the partners of internal supersymmetries:

$$
\left[M_{\mu \bar{I}}, Q_{\alpha}\right]=\frac{1}{2} \mathrm{i}\left(\sigma^{\mu} \bar{S}\right)_{\bar{I} \alpha}, \quad\left[M_{\mu \bar{I}}, S_{J \alpha}\right]=0 .
$$

Finally, one has

$$
\left[R_{I}, Q_{\alpha}\right]=S_{I \alpha}, \quad\left[R_{I}, S_{J \alpha}\right]=0,
$$

for the rescaled off-diagonal R-symmetries. Note that all rescaled, non-linearly realized generators transform in the fundamental of $\mathrm{SU}(3)$, and form a sequence analogous to (3.8) under conventional supersymmetry. In addition this algebra allows for a $\mathrm{U}(1)$ acting on $Q$.

The superspace action (3.13) can easily be extended to

$$
\Phi^{I}\left(\bar{D}_{\dot{\alpha}} \partial_{\mu} \bar{\Phi}^{\bar{I}} \bar{\sigma}_{\nu}^{\dot{\alpha} \alpha} D_{\alpha} \partial_{\rho} \Phi^{J}\right) \epsilon^{\mu \nu \rho \sigma} \partial_{\sigma} \bar{\Phi}^{\bar{J}}
$$

This is the unique generalization of the quartic invariant for a single superfield that is compatible with the $\mathrm{U}(1) \times \mathrm{U}(\mathcal{N})$ symmetry. Its invariance under the non-linearly realized internal supersymmetry can be seen in a very analogous manner as the discussion in section 3.

Despite the absence of linear supersymmetry representations without higher spins, there are analogous superalgebras in dimensions higher than ten, allowing for similar contractions. In this manner one can obtain the $\mathcal{N}$-extended generalization of the $D=6$ and $D=10$ results for arbitrary $\mathcal{N}$. Since the resulting algebras do not have any linear representations, and can be realized solely on spinors, this does not contradict the common statements that global supersymmetry has $\mathcal{N} \leq 4$ in $D=4$.

\section{Conclusions}

We have discussed the existence of a fermionic symmetry akin to bosonic internal symmetries. Being spontaneously broken, its Goldstino mode transforms with a constant fermionic parameter, reminiscent of Goldstone bosons for internal symmetries. This fermionic extension of Poincaré is inequivalent to conventional supersymmetry and instead can be constructed from İnönü-Wigner contractions of superalgebras, starting either in four or 
in higher dimensions. The latter case leads to linearly supersymmetric theories in which internal supersymmetry is the natural partner of the Galileon algebra.

Effective field theories have universal dynamics for the Goldstone modes, with particular signatures. In the case at hand, internal supersymmetry ensures the vanishing of its amplitudes in the soft limits; this is the fermionic analogon of the Adler zero, see the recent discussion [38]. An analysis analogous to $[4,5]$ could provide a periodic table of fermion effective field theories with soft limits.

We have demonstrated that no Wess-Zumino terms for internal supersymmetry exist in $D=4$ beyond the ordinary kinetic term. The fermion is therefore always derivatively coupled in absence of coupling to different fields. However, the supersymmetric Galileons of [33] provide an interaction which is nonetheless free of Ostrogradsky ghosts. It appears that the higher-dimensional origin of these constructions plays an important role in eliminating possible ghost degrees of freedom, as this particular realization of supersymmetric Galileon is shown here to satisfy a contracted higher-dimensional symmetry algebra. This connection was found long ago in the uncontracted case [39] and appears to be the same in the contracted case $[32,33]$.

All degrees of freedom considered in this paper are Goldstone modes, whose dynamics can be characterized in terms of a small number of coupling coefficients (in contrast to generic supersymmetry theories with e.g. Kähler and superpotentials). Important questions that we leave for future work include the coupling to matter, e.g. in $\mathcal{N}=1$ superspace. Another generalization involves other multiplets than the chiral superfield $\Phi$; for instance, it would be interesting to investigate the relation between the real linear superfield and the $D=5$ superalgebra. Because the real linear contains only a single scalar field, the $D=5$ translations and Galilean transformations can be realized on it while maintaining the constraints on the superfield. This might shed light on the higher-dimensional origin of supersymmetric theories based on a real Galileon scalar considered in [40] and/or the fermionic rescaling of [41].

The universal nature of Goldstone dynamics provides for a strong motivation to further elucidate these matters.

\section{Acknowledgments}

We are grateful to F. Farakos, R. Kallosh, R. Klein and D. Stefanyszyn for stimulating discussions, and in particular S. Garcia-Saenz for pointing out the Fierz identity for the putative quartic fermion interaction. The work of YY is supported by SITP and by the US National Science Foundation grant PHY-1720397. PW acknowledges the Dutch funding agency Netherlands Organisation for Scientific Research (NWO) for financial support. YY is grateful to the University of Groningen for the hospitality when this work was initiated.

Open Access. This article is distributed under the terms of the Creative Commons Attribution License (CC-BY 4.0), which permits any use, distribution and reproduction in any medium, provided the original author(s) and source are credited. 


\section{References}

[1] S.R. Coleman, J. Wess and B. Zumino, Structure of phenomenological Lagrangians. 1., Phys. Rev. 177 (1969) 2239 [INSPIRE].

[2] C.G. Callan Jr., S.R. Coleman, J. Wess and B. Zumino, Structure of phenomenological Lagrangians. 2., Phys. Rev. 177 (1969) 2247 [InSPIRE].

[3] D.V. Volkov, Phenomenological Lagrangians, Fiz. Elem. Chast. Atom. Yadra 4 (1973) 3.

[4] C. Cheung, K. Kampf, J. Novotny and J. Trnka, Effective Field Theories from Soft Limits of Scattering Amplitudes, Phys. Rev. Lett. 114 (2015) 221602 [arXiv:1412.4095] [INSPIRE].

[5] C. Cheung, K. Kampf, J. Novotny, C.-H. Shen and J. Trnka, A Periodic Table of Effective Field Theories, JHEP 02 (2017) 020 [arXiv:1611.03137] [INSPIRE].

[6] A. Padilla, D. Stefanyszyn and T. Wilson, Probing Scalar Effective Field Theories with the Soft Limits of Scattering Amplitudes, JHEP 04 (2017) 015 [arXiv: 1612.04283] [INSPIRE].

[7] C. de Rham and A.J. Tolley, DBI and the Galileon reunited, JCAP 05 (2010) 015 [arXiv:1003.5917] [INSPIRE].

[8] K. Hinterbichler, M. Trodden and D. Wesley, Multi-field galileons and higher co-dimension branes, Phys. Rev. D 82 (2010) 124018 [arXiv:1008.1305] [INSPIRE].

[9] G. Goon, K. Hinterbichler, A. Joyce and M. Trodden, Galileons as Wess-Zumino Terms, JHEP 06 (2012) 004 [arXiv: 1203.3191] [INSPIRE].

[10] G.R. Dvali, G. Gabadadze and M. Porrati, 4-D gravity on a brane in 5-D Minkowski space, Phys. Lett. B 485 (2000) 208 [hep-th/0005016] [INSPIRE].

[11] K. Hinterbichler, Theoretical Aspects of Massive Gravity, Rev. Mod. Phys. 84 (2012) 671 [arXiv:1105.3735] [INSPIRE].

[12] C. Deffayet, G. Esposito-Farese and A. Vikman, Covariant Galileon, Phys. Rev. D 79 (2009) 084003 [arXiv:0901.1314] [INSPIRE].

[13] S. Peirone, N. Frusciante, B. Hu, M. Raveri and A. Silvestri, Do current cosmological observations rule out all Covariant Galileons?, Phys. Rev. D 97 (2018) 063518 [arXiv: 1711.04760] [INSPIRE].

[14] D.V. Volkov and V.P. Akulov, Is the Neutrino a Goldstone Particle?, Phys. Lett. B 46 (1973) 109 [INSPIRE].

[15] E.A. Ivanov and A.A. Kapustnikov, General Relationship Between Linear and Nonlinear Realizations of Supersymmetry, J. Phys. A 11 (1978) 2375 [INSPIRE].

[16] E.A. Ivanov and A.A. Kapustnikov, The nonlinear realization structure of models with spontaneously broken supersymmetry, J. Phys. G 8 (1982) 167 [INSPIRE].

[17] C. Deffayet, A.E. Gümrükçüoglu, S. Mukohyama and Y. Wang, A no-go theorem for generalized vector Galileons on flat spacetime, JHEP 04 (2014) 082 [arXiv:1312.6690] [INSPIRE].

[18] R. Kallosh, Volkov-Akulov theory and D-branes, hep-th/9705118 [INSPIRE].

[19] R. Kallosh, A. Karlsson and D. Murli, Origin of Soft Limits from Nonlinear Supersymmetry in Volkov-Akulov Theory, JHEP 03 (2017) 081 [arXiv:1609.09127] [INSPIRE].

[20] R. Haag, J.T. Lopuszanski and M. Sohnius, All Possible Generators of Supersymmetries of the s Matrix, Nucl. Phys. B 88 (1975) 257 [inSPIRE]. 
[21] S.R. Coleman and J. Mandula, All Possible Symmetries of the S Matrix, Phys. Rev. 159 (1967) 1251 [INSPIRE].

[22] J. Bagger and A. Galperin, A new Goldstone multiplet for partially broken supersymmetry, Phys. Rev. D 55 (1997) 1091 [hep-th/9608177] [INSPIRE].

[23] J. Bagger and A. Galperin, The tensor Goldstone multiplet for partially broken supersymmetry, Phys. Lett. B 412 (1997) 296 [hep-th/9707061] [INSPIRE].

[24] M. Roček and A.A. Tseytlin, Partial breaking of global D=4 supersymmetry, constrained superfields and three-brane actions, Phys. Rev. D 59 (1999) 106001 [hep-th/9811232] [INSPIRE].

[25] F. Gonzalez-Rey, I.Y. Park and M. Roček, On dual 3-brane actions with partially broken $N=2$ supersymmetry, Nucl. Phys. B 544 (1999) 243 [hep-th/9811130] [INSPIRE].

[26] B. Bellazzini, Softness and amplitudes' positivity for spinning particles, JHEP 02 (2017) 034 [arXiv: 1605.06111] [INSPIRE].

[27] D. Liu, A. Pomarol, R. Rattazzi and F. Riva, Patterns of Strong Coupling for LHC Searches, JHEP 11 (2016) 141 [arXiv: 1603.03064] [INSPIRE].

[28] B. Bellazzini, F. Riva, J. Serra and F. Sgarlata, The other effective fermion compositeness, JHEP 11 (2017) 020 [arXiv: 1706.03070] [INSPIRE].

[29] H. Abe, Y. Sakamura and Y. Yamada, $N=1$ superfield description of vector-tensor couplings in six dimensions, JHEP 04 (2015) 035 [arXiv: 1501.07642] [INSPIRE].

[30] K. Hinterbichler and A. Joyce, Hidden symmetry of the Galileon, Phys. Rev. D 92 (2015) 023503 [arXiv: 1501.07600] [INSPIRE].

[31] J. Khoury, J.-L. Lehners and B. Ovrut, Supersymmetric $P(X, \phi)$ and the Ghost Condensate, Phys. Rev. D 83 (2011) 125031 [arXiv:1012.3748] [INSPIRE].

[32] M. Koehn, J.-L. Lehners and B. Ovrut, Supersymmetric cubic Galileons have ghosts, Phys. Rev. D 88 (2013) 023528 [arXiv: 1302.0840] [INSPIRE].

[33] F. Farakos, C. Germani and A. Kehagias, On ghost-free supersymmetric galileons, JHEP 11 (2013) 045 [arXiv: 1306.2961] [inSPIRE].

[34] J.M. Queiruga, Supersymmetric galileons and auxiliary fields in 2+1 dimensions, Phys. Rev. D 95 (2017) 125001 [arXiv: 1612.04727] [INSPIRE].

[35] H. Motohashi, K. Noui, T. Suyama, M. Yamaguchi and D. Langlois, Healthy degenerate theories with higher derivatives, JCAP 07 (2016) 033 [arXiv:1603.09355] [INSPIRE].

[36] R. Klein and D. Roest, Exorcising the Ostrogradsky ghost in coupled systems, JHEP 07 (2016) 130 [arXiv:1604.01719] [INSPIRE].

[37] R. Kimura, Y. Sakakihara and M. Yamaguchi, Ghost free systems with coexisting bosons and fermions, Phys. Rev. D 96 (2017) 044015 [arXiv: 1704.02717] [INSPIRE].

[38] R. Kallosh, Nonlinear (Super)Symmetries and Amplitudes, JHEP 03 (2017) 038 [arXiv: 1609.09123] [INSPIRE].

[39] J. Hughes, J. Liu and J. Polchinski, Supermembranes, Phys. Lett. B 180 (1986) 370 [INSPIRE].

[40] J. Khoury, J.-L. Lehners and B.A. Ovrut, Supersymmetric Galileons, Phys. Rev. D 84 (2011) 043521 [arXiv:1103.0003] [INSPIRE].

[41] K. Kamimura and S. Onda, Contractions of AdS brane algebra and superGalileon Lagrangians, J. Math. Phys. 54 (2013) 062503 [arXiv:1303.5506] [INSPIRE]. 